\title{
Reply to the correspondence letter by Se Jin Park et al. What dose of intravenous immunoglobulin should be administered in Kawasaki disease with suspected systemic capillary leak syndrome?
}

\author{
Farah Thabet
}

Received: 26 September 2011 / Accepted: 4 October 2011 /Published online: 19 October 2011

(C) Springer-Verlag 2011

We thank Dr. Se Jin Park and collaborators for their interesting informations and comments provided as reaction to our recent paper [3]. They mainly rise two points: (1) the possibility of a systemic capillary leak syndrome (SCLS) because of persistent hypoalbuminemia. (2) The recommendation to use higher dose of intravenous immunoglobulin in suspected SCLS associated with Kawasaki disease to prevent coronary artery aneurysm, as the author understood that Thabet et al. administered a moderate dose $(1 \mathrm{~g} / \mathrm{kg})$ of intravenous immunoglobulin.

The pathogenesis of Kawasaki disease is not yet totally established. One of the possible mechanisms could be vascular leakage associated with vasculitis as suggested by the study of Terai et al. [2]. Vascular endothelial growth factor was found to be elevated and inversely related to serum albumin in both resistant form of Kawasaki disease and SCLS. Yet, the patient we described had persistent hypoalbuminemia suggesting a certain degree of vascular leakage; it cannot be considered as SCLS as this entity is well defined by an association of hypoalbuminemia, hemoconcentration documented by increase in hemoglobin and hematocrit, and shock due to vascular collapse [1]. The patient's hemoglobin was not increased neither was the hematocrit.

Furthermore the patient received $1 \mathrm{~g} / \mathrm{kg}$ of intravenous immunoglobulin for 2 days (total dose $=2 \mathrm{~g} / \mathrm{kg}$ ) and not a moderate dose of $1 \mathrm{~g} / \mathrm{kg}$. Despite receiving a full dose of intravenous immunoglobulin, he developed coronary artery dilatation. Kawasaki disease shock syndrome is more likely to be intravenous immunoglobulin resistant and requires second line of treatment.

\section{References}

1. Druey KM, Greipp PR (2010) Narrative review: Clarkson diseasesystemic capillary leak syndrome. Ann Intern Med 153:90-98

2. Terai M, Honda T, Yasukawa K et al (2003) Prognostic impact of vascular leakage in acute Kawasaki disease. Circulation 108:325330

3. Thabet F, Bafaqih H, Al-Mohaimeed S et al (2011) Shock: an unusual presentation of Kawasaki disease. Eur J Pediatr 170:941943
F. Thabet $(\bowtie)$

Riyad Military Hospital,

Riyad, Saudi Arabia

e-mail: thabetfarah@yahoo.fr 